\title{
ReaR

\section{Valoración hemodinámica del paciente con shock séptico ¿Termodilución o ecocardiografía?}

Artículo original: Vignon P, Begot E, Mari A, Silva S, Chimot L, Delour P et al. Hemodynamic Assessment of Patients With Septic Shock Using Transpulmonary Thermodilution and Critical Care Echocardiography A Compara-tive Study CHEST 2018; 153(1):55-64 (PubMed) (HTML) (PDF)

Paz Martín D (1) Serna Gandía MB (2).

(1) Complejo Hospitalario de Toledo.

(2) Hospital Marina Salud Denia.

\section{Resumen}

El shock séptico representa una de las principales causas de fracaso circulatorio agudo en las Unidades de Cuidados Intensivos.

Tanto las técnicas de monitorización hemodinámica basadas en la termodilución (TPT), como la ecocardiografía (CCE) son ampliamente utilizadas en la monitorización de pacientes críticos con shock. Sin embargo, hasta la fecha, ambas modalidades de monitorización no han sido suficientemente comparadas.

El objetivo del presente trabajo fue evaluar el grado de concordancia en la interpretación de los parámetros cuantitativos derivados de la monitorización con TPT y CCE en una cohorte de pacientes con shock séptico ventilados mecánicamente.

\section{Introducción}

El shock séptico representa una de las principales causas de fracaso circulatorio agudo en las Unidades de Cuidados Intensivos.

Tanto las técnicas de monitorización hemodinámica basadas en la termodilución (TPT), como la ecocardiografía (CCE) son ampliamente utilizadas en la monitorización de pacientes críticos con shock. Sin embargo, hasta la fecha, ambas modalidades de monitorización no han sido suficientemente comparadas.

El objetivo del presente trabajo fue evaluar el grado de concordancia en la interpretación de los parámetros cuantitativos derivados de la monitorización con TPT y CCE en una cohorte de pacientes con shock séptico ventilados mecánicamente.

\section{Introducción}

El shock séptico (SS) representa una de las principales causas de fracaso circulatorio agudo en las Unidades de Cuidados Intensivos (UCI). Patofisiologicamente, la sepsis se caracteriza por vasoplejia con pérdida del tono arterial, venodilatación con secuestro de sangre en el compartimento venoso y cambios en la función ventricular, como la disminución de la distensibilidad cardíaca y la respuesta a los aumentos de precarga (1).

Tanto las técnicas de monitorización hemodinámica basadas en la termodilución (TPT), como la ecocardiografía (CCE) son ampliamente utilizadas en la monitorización de 
pacientes críticos con shock. Sin embargo, hasta la fecha, ambas modalidades de monitorización no han sido suficientemente comparadas. El objetivo del presente trabajo fue evaluar el grado de concordancia en la interpretación de los parámetros cuantitativos derivados de la monitorización con TPT y CCE en una cohorte de pacientes con SS ventilados mecánicamente.

\section{Material y Métodos}

Estudio descriptivo prospectivo realizado durante 36 meses en 5 UCIs francesas.

Población de estudio: pacientes en SS que precisaban ventilación mecánica, perfectamente adaptados al ventilador, en ritmo sinusal y con necesidad de monitorización hemodinámica avanzada. El SS se definió como hipotensión mantenida que requería vasopresores debido a una probable infección, a pesar de una adecuada carga de volumen, asociada con signos clínicos de hipoperfusión (livideces, encefalopatía, oliguria durante más de 2 h), que fue biológicamente confirmada ( $\mathrm{pH}<7,38$, déficit de bases $>-5 \mathrm{mmol} / \mathrm{L}$, lactato $>2 \mathrm{mmol} / \mathrm{L}$ o saturación venosa central de oxígeno $<70 \%)(2,3)$.

Fueron excluidos los pacientes menores de 18 años, las embarazadas, aquellos con ritmo cardiaco distinto del sinusal, los que presentaban contraindicaciones para la inserción de catéteres femorales o eco transesofágico, los pacientes moribundos o los ya incluidos en el estudio en algún momento previo.

Valoración hemodinámica: fue realizada por dos investigadores que no eran responsables directos del manejo del paciente, empleando de forma aleatoria TPT (PICCO plus) o ultrasonidos. Ambos estudios se realizaron de forma consecutiva sin realizar cambios terapéuticos entre ellos. Posteriormente cada investigador interpretó de forma independiente el perfil hemodinámico y propuso una estrategia terapéutica de acuerdo a un algoritmo predefinido (Fig. 1). Cada investigador tuvo acceso a la misma información clínica como antecedentes personales o resultados de laboratorio, pero no conocieron los detalles de la evaluación hemodinámica realizada con la otra técnica.

Los resultados de ambos estudios, así como las propuestas terapéuticas, se trasladaron al médico a cargo del paciente. En caso de discordancia en las propuestas, la estrategia a seguir fue decidida por el responsable directo del paciente.

Seguimiento de los pacientes: se realizó en cada sujeto un seguimiento durante 6 horas, evaluando el aclaramiento de lactato, el desarrollo de eventos adversos (bradicardia < 701pm, arritmias, edema agudo de pulmón). Se consideró aclaramiento de lactato cuando el nivel disminuía al menos un $10 \%$ o a niveles inferiores de $2 \mathrm{mmol} / \mathrm{L}$.

Los parámetros cuantitativos fueron comparados usando el test de la $\mathrm{T}$ de Student y las proporciones mediante la Chi cuadrado o el Test exacto de Fisher según fuera conveniente.

Se consideraron estadísticamente significativos los niveles de $\mathrm{p}<0,05$. El grado de concordancia entre las interpretaciones a pie de cama de los perfiles hemodinámicos fueron explorados mediante tablas de contingencia. 


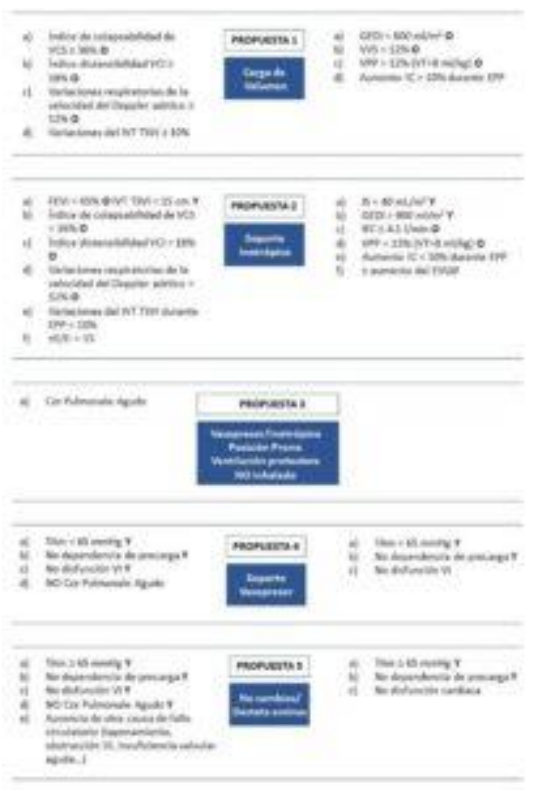

Fig 1. Algoritmo terapéutico según perfiles hemodinámicos

Resultados principales:

De los 234 pacientes consecutivos, 85 (36\%) presentaron criterios de exclusión y 12 de los 149 restantes finalmente no fueron incluidos en el estudio por diferentes motivos. Un total de 137 pacientes fueron analizados (71 hombres, edad media $61 \pm 15$ años, SAPS $58 \pm 18$, SOFA $10 \pm 3$ ).

Las causas más frecuentes de SS fueron la neumonía y la infección abdominal, y pudo documentarse microorganismo responsable en 122 pacientes (89\%).

Todos los sujetos recibían catecolaminas en el momento de inclusión. El tiempo de retraso entre las dos valoraciones hemodinámicas fue de $19 \pm 17 \mathrm{~min}$.

Mediante el empleo de CCE se identificaron 5 pacientes $(4 \%)$ con core pulmonale agudo (CPA).

Un total de 4 pacientes presentaron criterios de carga de volumen cuando se analizaron con CCE frente a 11 con TPT, y se sugirió inicio de terapia inotrópica en 10 vs 8 pacientes respectivamente.

Las interpretaciones entre TPT y CCE fueron concordantes en 87 de 132 pacientes $(66 \%)$ sin CPA, resultando en un moderado nivel de concordancia (kappa, 0.48; IC95\% 0.37-0.60). Las recomendaciones de actitudes terapéuticas de los expertos que participaban en el estudio coincidieron en 100 de 129 pacientes sin CPA $(77,5 \%)$, resultando en una buena concordancia intertécnica (kappa, 0.66; IC95\% 0.55-0.77).

En presencia de propuestas terapeuticas no concordantes derivadas de los estudios hemodinámicos realizados, el médico responsable decidió guiarse preferiblemente por los resultados de la ecografía (30 de 45 pacientes, 67\%), seguido de la interpretación de la TPT en 13 pacientes. En 2 pacientes (4\%) no se siguió ninguna de las propuestas.

Además, la CCE permitió identificar en 8 de 29 pacientes la causa potencial de las discrepancias entre las dos técnicas.

En 5 pacientes se evidenció valvulopatía izquierda severa, en 2 obstrucción dinámica del tracto de salida del ventrículo izquierdo y en otros 2, gastos cardiacos muy bajos. Se produjo aclaramiento de lactato a las 6 horas en 87 de 129 pacientes (69\%) sin CPA. La proporción fue similar independientemente del grado de concordancia en las interpretaciones con las dos técnicas; (55 de 84 [65\%] en el caso de concordancia vs 32 de 45 en caso de no concordancia [71\%], $\mathrm{P}=0,55)$. De igual modo, tampoco se observaron diferencias estadísticamente significativas en función de la concordancia en cuanto a las complicaciones o la mortalidad en UCI (29 de 87 [36\%] vs 13 de 45 [29\%], $\mathrm{P}=0.60$ ) o a 28 días (31 de 87 [36\%] vs 16 de 45 [36\%], $\mathrm{P}=0.99)$. 


\section{Discusión}

En este estudio, que compara la monitorización con TPT y con CCE, se observó una coincidencia en la interpretación del perfil hemodinámico en 2 de cada 3 casos. La respuesta a precarga fue el factor terapéutico más relevante, suponiendo un $50 \%$ de los casos y la administración de inotrópicos un $26 \%$. Siendo ambos resultados similares a lo publicado hasta la fecha (4). Los pacientes ya habían sido reanimados con fluidoterapia $y$ vasopresores, por lo que en una cuarta parte no hubo ningún cambio terapéutico propuesto.

Las discrepancias entre la TPT y la CCE fueron identificadas en un $43 \%$ de los casos mediante ecocardiografía, siendo el CPA la causa más frecuente.

El CPA es la forma más grave del fallo del ventrículo derecho y su identificación por TPT está infraestimada, ofreciendo además un patrón de respuesta a precarga positiva (aumento de la variabilidad del volumen sistólico), que puede llevar a una administración deletérea de volumen.

En estos casos, la CCE sí es capaz de detectar los falsos positivos. Por tanto, los autores de esta lectura crítica consideramos que la CCE debe ser la primera exploración a realizar en pacientes inestables con shock séptico y distrés asociado para detectar pacientes con CPA. No sólo en primer lugar, sino también siempre que se sospeche un fallo cardiaco, que puede aparecer en cualquier momento del proceso del shock séptico (5).

En otros 8 pacientes se encontraron valvulopatías severas (insuficiencia mitral y aórtica), obstrucción dinámica en el tracto de salida del ventrículo izquierdo y un índice cardiaco bajo. Todos ellos habrían interferido en el algoritmo de la TPT y precisaron de la CCE para ser diagnosticados. Es cierto que el aclaramiento de lactato no se vio influido por la concordancia de ambas técnicas, y que no hubo impacto a corto plazo en la eficacia y tolerancia de las actuaciones terapéuticas derivadas de ambas técnicas, ni en las complicaciones observadas. En este aspecto es preciso reseñar que el limitado tiempo de seguimiento de cada paciente no permite sacar conclusiones sobre el impacto de cada una de las técnicas a lo largo de todo el cuadro séptico.

\section{Conclusión}

Ambas técnicas se pueden utilizar con seguridad en el paciente inestable. La concordancia en la interpretación del perfil hemodinámico de esta cohorte de pacientes con ambas técnicas es moderada.

La CCE permite un diagnóstico inicial de la situación cardiológica del paciente $\mathrm{y}$, lo que es más importante, identifica la causa de discrepancias entre ambas técnicas en cerca de la mitad de los casos. Consideramos que la CCE debe ser utilizada siempre en primer lugar, dejando a la TPT como monitorización del gasto cardiaco e índices derivados, de forma continua. De este modo, ambos dispositivos resultan complementarios más que excluyentes.

\section{Bibliografía}

- Monitorización hemodinámica y fluidoterapia en la sepsis. D. Paz Martín, R. Vicho Pereira, MB Serna Gandía, En 50 Preguntas clave en Sepsis. Ed. Permamyer 201

- Dellinger RP, Levy MM, Rhodes A,Annane D, Gerlach H, Opal SM et al. Surviving Sepsis Campaign: international guidelines for management of severe sepsis and septic shock: 2012. Crit Care Med. 2013;41(2):580-637. (PubMed) 
- Bouferrache K, Amiel JB, Chimot L, Caille V, Charron $\mathrm{C}$, Vignon $\mathrm{P}$ et al. Initial resuscitation guided by the Surviving Sepsis Campaign recommendations and early echocardiographic assessment of hemodynamics in intensive care unit patients: a pilot study. Crit Care Med. 2012;40(10):2821-2827. (PubMed)

- Michard F, Teboul JL. Predicting fluid responsiveness in ICU patients: a critical analysis of the evidence. Chest. 2002;121(6):2000-8.(PubMed)

- Pulido JA, Afessa B, Masaki M, Yuasa T, Gillespie S, Herasevich $\mathrm{V}$ et al. Clinical Spectrum, Frequency, and Significance of Myocardial Dysfunction in Severe Sepsis and Septic
Shock. Mayo Clin Proc. 2012; 87(7): 620-628. ( $\underline{\text { PubMed }}$ ( $\underline{\text { HTML) }}$ ( $\underline{\text { PDF }})$

Correspondencia al autor

Daniel Paz Martín

dpaz@anestesiar.org

MD PhD, EDAIC, EDIC. Jefe de Sección Anestesiología y Reanimación.

Complejo Hospitalario de Toledo.

Sección de Cuidados Intensivos de la SEDAR

Aceptado para blog en agosto de 2018. 